\title{
Role of tumor necrosis factor-like weak inducer of apoptosis/ fibroblast growth factor-inducible molecule 14 pathway in lupus nephritis
}

\author{
Miriam R. Bekhit ${ }^{a}$, Nadia S. Kamel ${ }^{a}$, Manal O. Mohammed ${ }^{a}$,
} Nouran M. Abaza ${ }^{\text {, Somia A. Saad El Din }}{ }^{b}$

aPhysical Medicine, Rheumatology and Rehabilitation Department, 'Department of Pathology, Faculty of Medicine, Ain Shams University, Cairo, Egypt

Correspondence to Miriam RaafatSadekBekhit, MSc, Physical medicine, rheumatology and rehabilitation department, Ain Shams University,Cairo, Egypt. Tel: 01001088623; e-mail: miriambekhit@gmail.com

Received 28 March 2018

Accepted 29 July 2018

Egyptian Rheumatology \& Rehabilitation 2018, 45:182-187

\begin{abstract}
Objectives
To evaluate urinary tumor necrosis factor-like weak inducer of apoptosis (UTWEAK) levels as well as renal fibroblast growth factor-inducible molecule 14 (Fn14) expression by immunohistochemistry in patients with systemic lupus erythematosus (SLE) to assess the possible role of TWEAK level as an indicator of lupus nephritis (LN) and its relation to disease activity as well as pathological $\mathrm{LN}$ classification.

Patients and methods

Urinary levels of TWEAK using enzyme-linked immunosorbent assay and chemical and immunological markers of SLE were measured in 30 patients with SLE and 15 age-matched and sex-matched apparently healthy controls. Patients with SLE were divided into two subgroups: 15 patients with LN and 15 without LN. Disease activity was assessed using systemic lupus erythematosus disease activity index SLE disease activity index (SLEDAI). Fn14 was examined in renal biopsies from LN group by immunohistochemistry.

Results

A significantly higher UTWEAK level was found in patients having SLE with LN compared with those without $L N$ and controls $(F=149.2, P<0.001)$. UTWEAK had a highly significant positive correlation with proteinuria $(r=0.755, P<0.001)$ and a significant positive correlation with tSLEDAI and $\mathrm{rSLEDAI}(r=0.217, P<0.037$ and $r=0.476, P<0.024$, respectively). UTWEAK had a significant negative correlation with anti-dsDNA titer, C3, and C4 $(r=-0.579, P<0.008 ; r=-0.456, P<0.011$; and $r=-0.552, P<0.002$, respectively). Fn14 expression was detected in mesangial and tubular cells, mainly proximal tubular cells, in patients with LN.

Conclusion
\end{abstract}

UTWEAK is a specific and sensitive biomarker for detection of active LN.

\section{Keywords:}

biomarker, lupus nephritis, systemic lupus erythematosus, tumor necrosis factor-like weak inducer of apoptosis/fibroblast growth factor-inducible molecule 14

Egypt Rheumatol Rehabil 45:182-187

(C) 2018 Egyptian Society for Rheumatology and Rehabilitation

$1110-161 \mathrm{X}$

\section{Introduction}

Systemic lupus erythematosus (SLE) is arguably the most serologically and clinically diverse multisystem autoimmune disease characterized by autoantibody production, immune complex formation, and immunologically mediated tissue injury [1].

Renal involvement is the most common and serious manifestation of SLE. Approximately $60 \%$ of the patients with SLE have renal abnormalities and $25-50 \%$ of the patients manifest clinical renal disease at the time of diagnosis [2].

The cytokine tumor necrosis factor (TNF)-like weak inducer of apoptosis (TWEAK), also known as TNF ligand superfamily member 12 , is a pleiotropic and multifunctional cytokine member of the TNF superfamily that regulates inflammatory pathways by inducing multiple cellular responses depending on the cell type and its microenvironment. It binds to its receptor, fibroblast growth factor-inducible molecule 14 (Fn14), stimulating a wide array of physiological processes, such as cellular proliferation, migration, survival, differentiation, and induction of apoptosis [3].

The role of TWEAK in promoting an inflammatory response, renal cell proliferation, apoptosis, vascular changes, and fibrosis suggests that TWEAK may play an active role in the kidney in the context of lupus nephritis (LN) [4].

Subsequent clinical studies have indicated the utility of urinary levels of TWEAK in the diagnosis of active LN

This is an open access journal, and articles are distributed under the terms of the Creative Commons Attribution-NonCommercial-ShareAlike 4.0 License, which allows others to remix, tweak, and build upon the work non-commercially, as long as appropriate credit is given and the new creations are licensed under the identical terms. 
[5] However, in these studies, the quantification of TWEAK was performed in patients who had already received immunosuppressive treatment, which implies a high possibility that the urinary expression of TWEAK in these patients was modified. As a result, the objective of our study was to determine the utility of urinary levels of TWEAK as a biomarker of lupus renal activity in recently diagnosed, previously untreated patients with SLE and its correlation as well as Fn14 expression with disease activity and pathological LN classification.

\section{Patients and methods}

The present study is a case-control one that included 30 patients who were recently diagnosed with SLE according to the Systemic Lupus International Collaborating Clinics criteria [6]. A written consent was taken from the patients to participate in this study according to the ethics committee recommendations (REC 270/2015). They were selected from inpatients and outpatients in University Hospitals. Moreover, 15 age-matched and sex-matched apparently healthy individuals were also included in the study and served as a control group. Written consent was obtained from all patients and controls after a full explanation of the study was provided. The selected patients were divided into two groups as follows: patients with SLE with renal involvement who also underwent kidney biopsy according to American College of Rheumatology criteria [7] and patients with SLE without any evidence of renal affection.

\section{Exclusion criteria}

Patients with diabetes mellitus, those with malignancies, those with other autoimmune diseases, or those with end-stage renal disease whether on hemodialysis or not, were excluded from the study.

Full history taking and thorough clinical examination were carried out for all patients. Laboratory investigations, including complete blood picture, erythrocyte sedimentation rate, C-reactive protein using the latex agglutination method, urine analysis, serum creatinine, blood urea nitrogen, serum albumin, serum complement factors $\mathrm{C} 3$ and $\mathrm{C} 4$, antinuclear antibody by immunofluorescence technique, antidsDNA, and protein quantification in $24 \mathrm{~h}$ urine, were performed for all patients.

Urinary TWEAK (uTWEAK) levels were measured by human TWEAK enzyme-linked immunosorbent assay kits (R\&D Systems, Minneapolis, MN, USA).
Assessment of the disease activity was done using the systemic lupus erythematosus disease activity index SLE disease activity index (SLEDAI), which is a validated model among experienced clinicians for global assessments of disease activity in patients with SLE [8].

Renal pathology was classified according to the revised International Society of Nephrology and Renal Pathology Society classification [7], and immunohistochemistry was performed on formalin-fixed paraffin-embedded renal sections, visualized with antibody for human antihuman Fn14 polyclonal antibody, supplied by R\&D Systems.

\section{Statistical analysis}

The statistical analysis was carried out by IBM SPSS statistics (version 17.0, 2010; IBM Corp., USA). The means and SDs or the medians and interquartile ranges were used to summarize the characteristics of the samples. Categorical variables were summarized with frequencies and percentages. Spearman's correlation coefficients were established with the continual values of TWEAK, histological findings, and the SLEDAI score, depending on the distribution of the variables. Receiver operating characteristic curves were calculated to establish sensitivity and specificity for diagnosis of renal activity owing to LN. $P$ value less than 0.05 was considered significant.

\section{Results}

This study included 30 patients with SLE, who were classified into two subgroups according to whether or not they had LN: group I and group II. Group I included 15 patients with LN, comprising 11 (73.33\%) females and four $(26.67 \%)$ males. Their ages ranged from 18.0 to 43.0 years, with a mean age of $29.7 \pm 8.8$ years. Their disease duration ranged from 1 to 14.0 months, with a median of 5 months. The uTWEAK level ranged from 40 to $75 \mathrm{pg} /$ $\mathrm{ml}$ (mean $\pm \mathrm{SD}, 51.0 \pm 8.9 \mathrm{pg} / \mathrm{ml}$ ). SLEDAI ranged from 19 to 35 .

Group II included 15 patients without LN, and they were all females (100\%). Their ages ranged from 21.0 to 45.0 years, with a mean age of $30.0 \pm 10.1$ years. Their disease duration ranged from 2 to 16.0 months, with a median of 4 months. The uTWEAK level ranged from 10 to $30 \mathrm{pg} / \mathrm{ml}$ (mean $\pm \mathrm{SD}, 16.7 \pm 5.2 \mathrm{pg} / \mathrm{ml})$. SLEDAI ranged from 4 to 21 .

All patients with LN (group I) underwent renal biopsy, and grading was done according to International Society of Nephrology and Renal Pathology Society 
Fig. 1
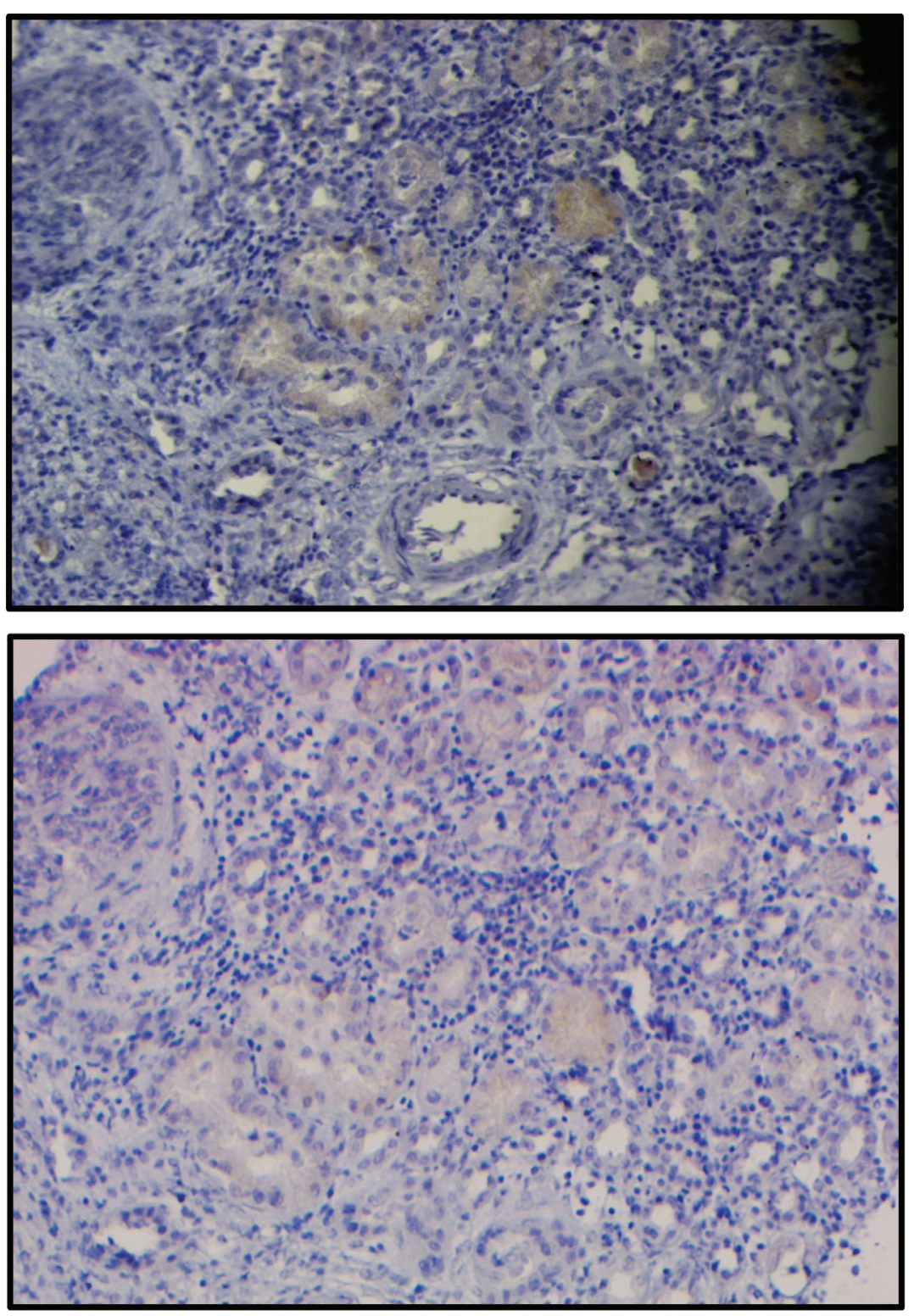

Different fields from same specimen ISN/RPS class IV-G (A/C), AI 18/24,

CI 1/12 showing mild to moderate Fn 14 expression intensity, mainly PCT $\times 200$.

Tubular Fn14 expression in a renal specimen from a patient with LN. Different fields from the same specimen ISN/RPS class IV-G (A/C), Al 18/ 24, and $\mathrm{Cl} 1 / 12$ showing mild to moderate Fn14 expression intensity, mainly PCT $\times 200$. Fn14, fibroblast growth factor-inducible molecule 14; ISN/RPS, International Society of Nephrology and Renal Pathology Society; LN, lupus nephritis.

classification. It was observed that three (20\%) were grade II glomerulonephritis (GN), three (20\%) were grade III GN, and nine (60\%) were grade IV GN. Fn14 expression was detected in mesangial and tubular cells, mainly proximal tubular cells, and it was associated with tubular atrophy and areas of interstitial fibrosis (Figs 1 and 2).

The clinical and laboratory data of the patients are shown in Tables 1 and 2. A significant difference was observed among the groups regarding uTWEAK $(P<0.001)$ (Table 3).
Correlation of uTWEAK with different studied parameters revealed a directly proportional correlation between the levels of UTWEAK and the tSLEDAI score and rSLEDAI score $(r=0.217, P=0.037$, and $r=0.476$, $P=0.024$, respectively); however, no correlation was observed between the levels of UTWEAK and extrarenal SLEDAI score $(r=0.340, \quad P=0.389)$. Additionally, a direct inverse correlation was documented between the levels of UTWEAK and the C3 $(r=-0.456, P=0.011)$ and $\mathrm{C} 4$ complement fractions $(r=-0.552, P=0.002)$ as well as anti-dsDNA titer $(r=-0.579, P=0.008)$. In contrast, the levels of 
Fig. 2

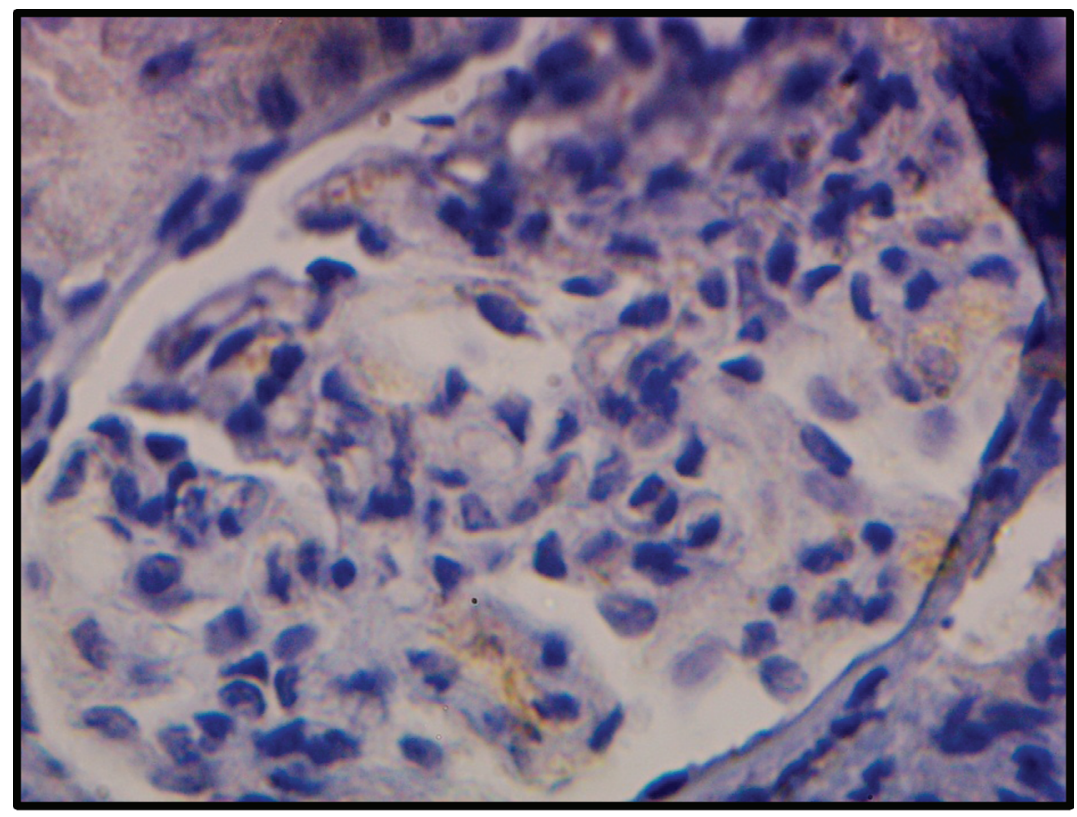

A specimen from ISN/RPS class II, AI 3/24, CI 3/12 showing mesangial moderate Fn14 expression $\times 400$.

Mesangial Fn14 expression in a renal specimen from a patient with LN. A specimen from ISN/RPS class II, AI 3/24, and CI 3/12, showing mesangial moderate Fn14 expression $\times 400$. Fn14, fibroblast growth factor-inducible molecule 14; ISN/RPS, International Society of Nephrology and Renal Pathology Society; LN, lupus nephritis.

Table 1 Frequency of the clinical data in patients with systemic lupus erythematosus

\begin{tabular}{lcc}
\hline Clinical manifestations & $\begin{array}{c}\text { Group I }(N=15) \\
{[n(\%)]}\end{array}$ & $\begin{array}{c}\text { Group II }(N=15) \\
{[n(\%)]}\end{array}$ \\
\hline Fever & $4(26.67)$ & $5(33.33)$ \\
Fatigue & $7(46.67)$ & $10(66.67)$ \\
Arthralgia & $12(80.00)$ & $10(66.67)$ \\
Malar rash & $12(80.00)$ & $11(73.33)$ \\
Photosensitivity & $10(66.67)$ & $10(66.67)$ \\
Alopecia & $8(53.33)$ & $11(73.33)$ \\
Mucosal ulcers & $10(66.67)$ & $12(80.00)$ \\
Headache & $2(46.67)$ & $3(20.00)$ \\
Cerebrovascular stroke & $0(0.00)$ & $2(13.33)$ \\
Pleurisy & $4(26.67)$ & $2(13.33)$ \\
Pleural effusion & $3(20.00)$ & $2(13.33)$ \\
Pericardial effusion & $2(13.33)$ & $1(6.67)$ \\
Raynaud's phenomenon & $7(46.67)$ & $5(33.33)$ \\
Recurrent abortions & $2(13.33)$ & $1(6.67)$ \\
\hline
\end{tabular}

proteinuria showed a directly proportional correlation with the values of uTWEAK $(r=0.755, P<0.001)$.

Using uTWEAK levels above $20 \mathrm{pg} / \mathrm{mg}$ as a cutoff point, the receiver operating characteristic curve analysis showed a sensitivity of $53.33 \%$ and a specificity of $100 \%$, with an area under the curve of 0.789 (95\% confidence interval, 0.642-0.896) (Fig. 3).

\section{Discussion}

Renal affection owing to SLE is a severe complication that has a direct effect on patient survival. The diagnosis of lupus nephropathy is based on clinical features, biochemical parameters, and immunological markers; however, no specific biomarker correctly defines renal activity for lupus [9].

In recent years, several studies have found that a disorder of the regulation of apoptosis is an important factor in SLE progress, such as TWEAK, and might play an important role in the pathogenesis of LN [10]. In this study, we analyzed the relationship between TWEAK and the renal activity of lupus to prove TWEAK as a marker of LN.

In our study, LN group showed a statistically significant higher uTWEAK levels compared with non-LN SLE group and normal healthy controls. Similar results were found by Schwartz et al. [11], Xuejing et al. [12], and El-Shehaby et al. [13].

We also found that uTWEAK levels were significantly positively correlated with tSLEDAI and rSLEDAI $(P<0.05)$. These findings suggest that the association of uTWEAK with active lupus is primarily dependent on the renal component of the score and does not depend on other systemic 
Table 2 Laboratory data in patients with systemic lupus erythematosus

\begin{tabular}{|c|c|c|c|c|}
\hline \multirow[t]{2}{*}{ Parameters } & \multicolumn{2}{|c|}{ Group I } & \multicolumn{2}{|c|}{ Group II } \\
\hline & Range & Mean \pm SD & Range & Mean \pm SD \\
\hline $\mathrm{Hb}(\mathrm{g} / \mathrm{dl})$ & $7.1-13.7$ & $9.9 \pm 2.0$ & $7-12.1$ & $9.0 \pm 1.3$ \\
\hline $\operatorname{RBC}\left(10^{6} / \mathrm{mm}^{3}\right)$ & $2.57-5.25$ & $3.7 \pm 0.8$ & $2.7-4.9$ & $3.6 \pm 0.5$ \\
\hline WBCs $\left(10^{3} / \mathrm{mm}^{3}\right)$ & $3.4-12$ & $7.7 \pm 3.0$ & $4.5-9.4$ & $7.0 \pm 1.3$ \\
\hline Platelets $\left(10^{3} / \mathrm{mm}^{3}\right)$ & $104-568$ & $259.9 \pm 124.6$ & $130-568$ & $248.6 \pm 116.3$ \\
\hline CRP $(\mathrm{mg} / \mathrm{l})$ & 6-48 & $44.5 \pm 38.0$ & 6-70 & $36.1 \pm 37.9$ \\
\hline ESR $(\mathrm{mm} / \mathrm{h})$ & $15-116$ & $61.9 \pm 39.2$ & $10-124$ & $47.2 \pm 43.6$ \\
\hline RBG (mg/dl) & $74-98$ & $82.7 \pm 10.7$ & $70-96$ & $77.3 \pm 11.2$ \\
\hline 2hPP (mg/dl) & $100-130$ & $112.1 \pm 9.1$ & $98-137$ & $120.5 \pm 13.0$ \\
\hline AST (IU/I) & 8-22 & $15.1 \pm 4.4$ & $8-23$ & $14.5 \pm 4.5$ \\
\hline ALT (IU/I) & $7-21$ & $14.1 \pm 4.5$ & $1-23$ & $12.5 \pm 6.8$ \\
\hline C3 (g/l) & $30-152$ & $91.2 \pm 45.8$ & $65-180$ & $126.3 \pm 36.8$ \\
\hline C4 (g/l) & $5-40$ & $24.7 \pm 12.2$ & $27-60$ & $36.7 \pm 8.4$ \\
\hline BUN (mg/dl) & $13-88$ & $50.1 \pm 22.3$ & $7-21$ & $13.5 \pm 4.3$ \\
\hline Creatinine (mg/dl) & $0.6-3.8$ & $1.9 \pm 0.8$ & $0.6-0.9$ & $0.7 \pm 0.1$ \\
\hline GFR (ml/min) & $30-126$ & $72.9 \pm 30.9$ & $115-170$ & $136.8 \pm 17.5$ \\
\hline Proteinuria $(\mathrm{mg} / 24 \mathrm{~h})$ & $0.521-6.47$ & $2.035 \pm 1.842$ & $0.002-0.096$ & $0.040 \pm 0.033$ \\
\hline$P / C$ ratio & $0.35-12$ & $4.6 \pm 4.5$ & $0.026-0.18$ & $0.1 \pm 0.50$ \\
\hline
\end{tabular}

2hPP, 2-hour postprandial glucose; ALT, alanine aminotransferase; AST, aspartate aminotransferase; BUN, blood urea nitrogen; CRP, Creactive protein; ESR, erythrocyte sedimentation rate; GFR, glomerular filtration rate; Hb, hemoglobin; RBC, red blood cell; RBG, random blood glucose; WBC, white blood cell.

Table 3 Comparison between studied groups regarding urinary tumor necrosis factor-like weak inducer of apoptosis levels

\begin{tabular}{lccccc}
\hline Groups & \multicolumn{2}{c}{ Urine TWEAK $(\mathrm{pg} / \mathrm{ml})$} & & \multicolumn{2}{c}{ ANOVA } \\
\cline { 2 - 3 } \cline { 5 - 6 } & Range & Mean \pm SD & & $F$ & $P$ value \\
\hline Group I & $40-75$ & $51.000 \pm 8.904$ & & 149.222 & $<0.001$ \\
Group II & $10-30$ & $16.667 \pm 5.233$ & & \\
Control & $10-20$ & $15.000 \pm 4.226$ & & \\
Tukey's test & & & & \\
Group I and group II & Group I and control & & $\begin{array}{c}\text { Group II and } \\
\text { control }\end{array}$ \\
& & & & 0.760 \\
\hline 0.001 & & $<0.001$ & & \\
\hline
\end{tabular}

ANOVA, analysis of variance; TWEAK, tumor necrosis factor-like weak inducer of apoptosis.

Fig. 3

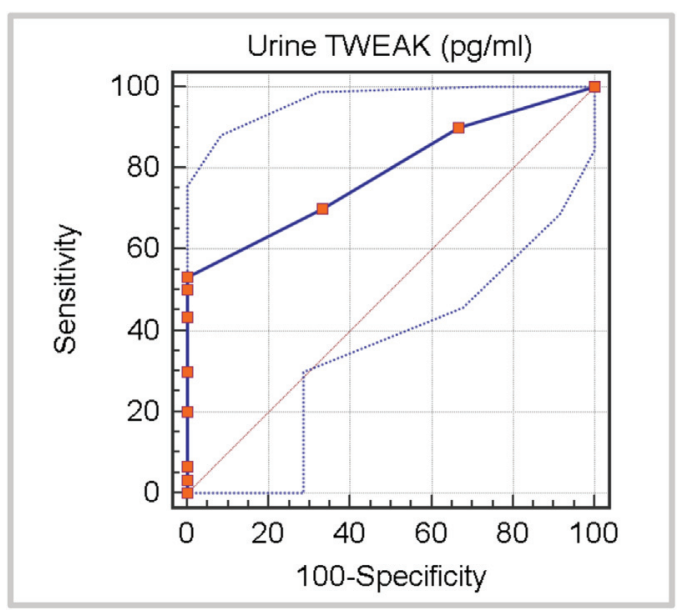

ROC curve analysis showing the diagnostic performance of urinary TWEAK. ROC, receiver operating characteristic; TWEAK, tumor necrosis factor-like weak inducer of apoptosis. parameters of the SLEDAI score. Similar results were found by Schwartz et al. [11] and El-Shehaby et al. [13].

Regarding 24-h urinary protein level, uTWEAK levels were significantly positively correlated with it $(P<0.01)$. Similar results were found by El-Shehaby et al. [13]. On the contrary, Schwartz et al. [11] did not find a significant correlation between UTWEAK and the degree of proteinuria. This may be owing to the higher mean rSLEDAI in our study of 13.9 compared with the lower mean rSLEDAI in their study of 4 , and the mean value of the 24-h urinary protein level in our study is 2.6 compared with the mean value of the 24-h urinary protein level in their study of 0.6 , reflecting that our patients had more active renal disease.

The underlying mechanisms why TWEAK relates to the development of proteinuria are unknown. However, experimental studies have shown that TWEAK activates the nuclear factor kappa-light-chainenhancer of activated B cells pathway to induce the expression of both soluble and membrane-bound inflammatory chemokines, including monocyte chemoattractant protein- 1 in podocytes, regulated on activation, normal $\mathrm{T}$ cell expressed and secreted, and $\mathrm{C}$ $\mathrm{X}-\mathrm{C}$ motif ligand 16 in a dose-dependent manner, and podocyte-derived monocyte chemoattractant protein-1 has been reported to promote motility and actin cytoskeleton rearrangement and decrease nephrin expression [14]. 
In an effort to explore the relationship between UTWEAK levels and the renal histology, we found that uTWEAK levels showed no differences between classes of $\mathrm{LN}$ on renal biopsy $(P=0.230)$. This indicates that UTWEAK detection cannot replace renal biopsy entirely in the diagnostic process. Similar results were found by Schwartz et al. [11] and Xuejing et al. [12]. This probably relates to the small number of patients studied who are further subgrouped into a number of histological classes, the inherent sampling error associated with renal biopsy, and the lack of a clear system to assess inflammatory disease activity at the tissue level [15].

However, the uTWEAK levels showed significantly positive correlations with the histological renal activity index $(P=0.007)$ but not the renal chronicity index $(P=0.644)$. This confirms that the elevation of uTWEAK level shows a stronger association with the renal disease activity. Similar results were found by El-Shehaby et al. [13] and Xuejing et al. [12].

TWEAK actions are regulated by the amount of receptor; a reduced Fn14 expression limits the actions of TWEAK in healthy tissues; however, under pathological conditions, the rapid and massive upregulation of this receptor sensitizes it to TWEAK actions [10]. In the present study, patients with LN showed higher levels of uTWEAK levels than patients without LN and controls $(P<0.001)$, and their renal biopsy showed Fn14 expression was detected in mesangial and tubular cells, mainly proximal tubular cells, and it was associated with tubular atrophy and areas of interstitial fibrosis.

The activation of the TWEAK/Fn14 inflammatory pathway contributes to the infiltration of macrophages and lymphocytes at the glomerular and tubular levels, direct cytotoxicity, and mesangial and tubular apoptosis. All these changes, added to the activation of the complement system and the deposit of immune complexes, accelerate kidney damage [16].

Several studies have provided data demonstrating the association of tissue mRNA expression of TWEAK and Fn14 in different diseases in both mouse models and human patients [17]. However, to our knowledge, this is the first study to explore the Fn14 expression by immunohistochemistry in renal biopsies from human patients with LN. Our preliminary data should be confirmed in a greater number of patients.

\section{Conclusion}

Our results demonstrated that uTWEAK is a sensitive biomarker for detection of LN in a sample of Egyptian patients, who were recently diagnosed and did not receive prior immunosuppressive treatment. However, performance of further studies on patients with $\mathrm{LN}$ is recommended to define its possible role in monitoring response to therapy as well as renal activity relapses.

\section{Financial support and sponsorship \\ Nil.}

\section{Conflicts of interest}

There are no conflicts of interest.

\section{References}

1 Mohan C, Putterman C. Genetics and pathogenesis of systemic lupus erythematosus and lupus nephritis. Nat Rev Nephrol 2015; 11:329-341.

2 Kwok SK, Tsokos GC. New insights into the role of renal resident cells in the pathogenesis of lupus nephritis. Korean J Intern Med 2018; 33:284-289.

3 Poveda J, Tabara LC, Fernandez-Fernandez B, Martin-Cleary C, Sanz AB, Selgas R, et al. TWEAK/Fn14 and non-canonical NF-kappaB signaling in kidney disease. Front Immunol 2013; 4:447-453.

4 Michaelson JS, Wisniacki N, Burkly LC, Putterman C. Role of TWEAK in lupus nephritis: a bench-to-bedside review. J Autoimmun 2012; 39:130-142.

$5 \mathrm{Xu}$ WD, Zhao Y, Liu Y. Role of the TWEAK/Fn14 pathway in autoimmune diseases. Immunol Res 2016; 64:44-50.

6 Petri M, Orbai AM, Alarcon GS, Gordon C, Merrill JT, Fortin PR, et al. Derivation and validation of the Systemic Lupus International Collaborating Clinics classification criteria for systemic lupus erythematosus. Arthritis Rheum 2012; 64 (8):2677-2686.

7 Hahn BH, McMahon M, Wilkinson A, Wallace WD, Daikh DI, FitzGerald J, et al. American College of Rheumatology Guidelines for screening, case definition, treatment and management of lupus nephritis. Arthritis Care Res 2012; 64:797-808.

8 Bombardier C, Gladman D, Urowitz M, Caron D. Derivation of the SLEDAI. A disease activity index for lupus patients. The committee on prognosis studies in SLE. Arthritis Rheum 2012; 35:630-640.

9 Reyes-Martinez F, Perez-Navarro M, Rodriquez-Matias A, Soto-Abraham V, Gutierrez-Reyes G, Medina-Avila Z, et al. Assessment of urinary TWEAK levels in Mexican patients with untreated lupus nephritis: an exploratory study. Nefrologia 2018; 38:152-160.

10 Sanz AB, Sanchez-Nino MD, Izquierdo MC, Moreno JA, Ucero AC, BenitoMartin A, et al. TWEAK, the facilitator of acute kidney injury. Nefrologia 2008; 28:587-592.

11 Schwartz N, Rubinstein T, Burkly LC, Collins CE, Blanco I, Su L, et al. Urinary TWEAK as a biomarker of lupus nephritis: a multicenter cohort study. Arthritis Res Ther 2009; 11:R143.

12 Xuejing Z, Jiazhen T, Jun L, Xiangqing X, Shuguang Y, Fuyou L. Urinary TWEAK Level as a Marker of Lupus Nephritis Activity in 46 Cases. $J$ Biomed Biotechnol 2012; 2012:359647.

13 El-Shehaby A, Darweesh H, El-Khatib M, Momtaz M, Marzouk S, ElShaarawy $\mathrm{N}$, et al. Correlations of urinary biomarkers, TNF-like weak inducer of apoptosis (TWEAK), osteoprotegerin (OPG), monocyte chemoattractant protein-1 (MCP-1), and IL-8 with lupus nephritis. J Clin Immunol 2011; 31:848-856.

14 Izquierdo MC, Sanz AB, Mezzano S, Blanco J, Carrasco S, Sanchez-Nino $\mathrm{MD}$, et al. TWEAK (tumor necrosis factor-like weak inducer of apoptosis) activates CXCL16 expression during renal tubulointerstitial inflammation. Kidney Int 2012; 81:1098-1107.

15 Dhaun N, Kluth DC. TWEAK: a novel biomarker for lupus nephritis. Arthritis Res Ther 2009; 11:133-135.

16 Gonzalez-Sanchez DA, Alvarez CM, Vasquez G, Gomez-Puerta JA. Role of TWEAK/Fn14 signalling pathway in lupus nephritis and other clinical settings. Nefrologia 2017; 37:118-125.

17 Sharif MN, Campanholle G, Nagiec EE, Wang J, Syed J, O'Neil SP, et al. Soluble Fn14 is detected and elevated in mouse and human kidney disease. PLoS One 2016; 11:e0155368. 